\title{
Construction of a novel IQA Function and Applications
}

\author{
B. Zhang \\ Dept. of Computer of \\ Chongqing Industry Polytechnic \\ College \\ Chongqing, China \\ e-mail: cqipcmail@163.com
}

\author{
Y. Li, T.T. Huang, X.F. Lv \\ College of Automatics of Chongqing \\ University of Posts and \\ Telecommulications \\ Chongqing, China
}

\author{
Z.X. Xie \\ Dept of Biomedical engineering of \\ Chongqing Medical University \\ Chongqing, China \\ e-mail: bmexxie@163.com
}

\begin{abstract}
The current image quality methods can only be able to assess quality degradation and can not be able to assess quality improvement after an image passing through a system. Here proposes a novel image quality assessment function (IQAF) based on the long term experiences. The IQAF is able to assess quality degradation as well as quality improvement after an image passing through a system. The IQAF can be able to assess single image quality as well as to compare multiple images quality. The $I Q A F$ is constructed by five visual quality parameters including averaging information entropy ( $A I E)$, averaging physical contrast level ( $A P C L)$, normalized neighborhood function $(N N F)$, average hierarchy factor $(A H F)$ and average bandwidth factor( $A B W F)$. The examples of $I Q A F$ applications were demonstrated finally.
\end{abstract}

Keywords-image quality assessment function (IQAF); average information entropy (AIE); average physical contrast level (APCL); normalized neighborhood function (NNF); average hierarchy factor (AHF); averaging bandwidth factor (ABWF)

\section{INTRODUCTION}

Currently, the methods of image quality assessment (IQA) mainly include the full reference image quality assessment (FR-IQA) and No reference image assessment (NR-IQA) [1-3]. Actually, FR-IQA, such as [2-3], is to assess the difference between objective image and priori reference image which is prior to be assumed as a standard image with the best quality, rater than really assesses the quality of an image. The assessment method always obtained an establishing value less than 1 , either an image whose quality is better than the reference image, e.g. an image that passed through an image enhancement system or an image whose quality is lower than the reference image, e.g. an image that passed through a noisy system. In a word, FR-IQA method can not be used to assess the image whose quality is improved after passing though an image quality enhancement system. This performance made its utilization to be limited. NR-IQA method is in exploring $[\mathbf{1}, \mathbf{3 - 8}]$ and there were a little articles published, especially in the color image field [1-3, 9-10].

In this paper, we constructed a novel image quality assessment function (IQAF) which is a product of averaging information entropy (AIE), averaging physical contrast level (APCL), normalized neighborhood function (NNF), average hierarchy factor (AHF) and average bandwidth factor $(\boldsymbol{A B W F})$. The $\mathbf{I Q A F}$ can assess that either image quality is better or is worse after an image passing though a system, either can compare the quality difference among multiple images or can assess a simple image quality [11], either can used in an active IQA or in a passive IQA.

\section{CONSTRUCTION OF IQAF}

\section{A. Construction of IQAF)}

Based on five visual quality parameters of an image, the $\mathbf{I Q A F}$ was constructed as follows.

$$
C A F=A I E * A P C L * N N F * A H F * A B W F
$$

where $\boldsymbol{C A F}$ is called as the comprehensive image quality assessment function. AIE is called averaging information entropy (AIE), APCL is called averaging physical contrast level, $N N F$ is called normalized neighborhood function, $\boldsymbol{A H F}$ is called average hierarchy factor and $\boldsymbol{A B W F}$ is called average bandwidth factor. The better the image quality, the bigger the IQAF value.

\section{B. Computation of AIE}

The AIE was computed as follows.

$$
A I E=\frac{1}{\sqrt{3}} \sqrt{\sum_{i=0}^{2} I E^{2}(i)}
$$

where,

$$
I E(i)=-\sum_{g=0}^{255} p(i, g) \log _{2} p(i, g), \quad i=0,1,2
$$

where $\boldsymbol{I E}(\boldsymbol{i})$ is the information entropy of each color component. Its unit is Ibit and can be called "information bit”. $\boldsymbol{i}=\mathbf{0 , 1 , 2}$ represents three color components of the red, the green and the blue. When $\boldsymbol{p}(\boldsymbol{i}, \boldsymbol{g})=\mathbf{0}$, let $\log _{2} \boldsymbol{p}(\boldsymbol{i}, \boldsymbol{g})=\mathbf{0}$. Any of color components has maximal information entropy, $I E(i)_{\max }$, of 8Ibit if a color spectrum possesses 256 spectral lines and uniform distribution(each of spectral lines has equal number of pixels).

\section{Computation of APCL}

The $\boldsymbol{A P C L}$ was computed as follows.

$$
A P C L=\frac{1}{\sqrt{3}} \sqrt{\sum_{i=0}^{2} A P C L^{2}(i)}, \quad i=0,1,2 \text { (Cbit) }
$$

where $\boldsymbol{A P C L}(\mathbf{i})$ is the average physical contrast level of each color component. Its unit is Cbit. $\mathbf{i}=\mathbf{0 , 1 , 2}$ represents three color components of the red, the green and the blue. The APCL(i) was computed as follows. 


$$
A P C L(i)=\frac{1}{M * N} \sum_{x=0}^{M-1} \sum_{y=0}^{N-1} \log _{2} C(i, x, y), \quad i=0,1,2 \text { (Cbit) }
$$

where $\boldsymbol{C}(\boldsymbol{i}, \boldsymbol{x}, \boldsymbol{y})$ is the contrast of each color component. Its unit is the luminance gradient (LG). $\mathbf{i}=\mathbf{0 , 1 , 2}$ represents three color components of the red, the green and the blue. The $\boldsymbol{C}(\boldsymbol{i}, \boldsymbol{x}, \boldsymbol{y})$ was called the image contrast and computed as follows.

$$
C(i, x, y)=\frac{1}{\sqrt{2}}|\vec{C}(i, x, y)|, \quad i=0,1,2
$$

where $\mathbf{i}=\mathbf{0 , 1 , 2}$ represents three color components of the red, the green and the blue. $\vec{C}(i, x, y)$ is the luminance gradient of each color component and was computed as follows.

$$
\vec{C}(i, x, y)=\frac{\partial L(i, x, y)}{\partial x} \vec{i}+\frac{\partial L(i, x, y)}{\partial y} \vec{j}, \quad i=0.1,2
$$

where $\boldsymbol{i}=\mathbf{0 , 1 , 2}$ represents three color components of the red, the green and the blue. $L(i, x, y)$ is the luminance of each color component at pixel with coordinates $\boldsymbol{x}$ and $\boldsymbol{y}$ and is measured directly from input image.

\section{Computation of NNF}

The normalized neighborhood function, $N \boldsymbol{N F}$, is computed as follows.

$$
N N F=f(A L)=1-a b s\left(A L-A L_{o p}\right) / A L_{o p}
$$

where abs (.) is called the absolute operater. $\boldsymbol{A} \boldsymbol{L}_{\boldsymbol{o p}}$ is called the optmal average luminance and its value is equal to $127.5[\mathbf{1 , 1 1}] . \boldsymbol{A L}$ is average luminance and was computed as follows.

$$
A L=\frac{1}{\sqrt{3}} \sqrt{\sum_{i=0}^{2} A L(i)}, \quad i=0,1,2
$$

where $\mathbf{i}=\mathbf{0 , 1 , 2}$ represents three color components of the red, the green and the blue. $\boldsymbol{A L}(\mathbf{i})$ is the average of each color components and was computed as follows.

$$
A L(i)=\frac{1}{M * N} \sum_{x=0}^{M-1} \sum_{y=0}^{N-1} L(i, x, y), \quad i=0,1,2
$$

where $\mathbf{i}=\mathbf{0 , 1 , 2}$ represents three color components of the red, the green and the blue. $L(i, x, y)$ is the luminance of each color component at pixel with coordinates $\boldsymbol{x}$ and $\boldsymbol{y}$ and is measured directly from input image.

\section{E. Computation of $\mathbf{A H F}$}

The average hierarchy factor of an image, $\boldsymbol{A H F}$, was defined as follows.

$$
A H F=\frac{1}{\sqrt{3}} \sqrt{\sum_{i=0}^{2} H F^{2}(i)} \quad i=0,1,2
$$

where $\mathbf{i}=\mathbf{0 , 1 , 2}$ represents three color components of the red, the green and the blue. $\boldsymbol{H F ( i )}$ is the hierarchy factor of each color components and was computed as follows.

$$
H F(i)=\text { Count }[P(i, 1, g)] / 256, \quad i=0,1,2
$$

where $\mathbf{i}=\mathbf{0 , 1 , 2}$ represents three color components of the red, the green and the blue. $\operatorname{Count}[\mathbf{P}(\mathbf{i}, \mathbf{1}, \mathbf{g})] / 256$ is called the count operator of spectral lines of chromatic spectra[1, 1112]. $\boldsymbol{P}(\boldsymbol{i}, \mathbf{1}, \boldsymbol{g})$ is a pixel number distribution function on chroma. The constant of 256 is the maximal probable spectral lines

\section{F. Computation of $\mathbf{A B W F}$}

The average bandwidth factor of an image, $\boldsymbol{A} \boldsymbol{B W F}$, was defined as follows.

$$
A B W F=\frac{1}{\sqrt{3}} \sqrt{\sum_{i=0}^{2} B W F^{2}(i)}, i=0,1,2
$$

where $\boldsymbol{i}=\mathbf{0 , 1 , 2}$ represents three color components of the red, the green and the blue. $\boldsymbol{B W F ( i )}$ is called the bandwidth factor of each color component of an image and computed as follows.

$$
B W F(i)=[\operatorname{Right}(i)-\operatorname{Left}(i)+1] / 256, \quad i=0,1,2
$$

where $\mathbf{i}=\mathbf{0 , 1 , 2}$ represents three color components of the red, the green and the blue. $\boldsymbol{L e f t}_{\boldsymbol{x}}[\bullet], \boldsymbol{R i g h t}_{\boldsymbol{x}}[\bullet]$ denote taking $\mathrm{x}$ coordinate value at the left boundary and at the right boundary of spectral lines of chromatic spectra of a color image $[\mathbf{1 , 1 1}$, respectively.

\section{APPLICATIONS OF IQAF}

\section{A. Comparison among the Qualities of the Images}

The bigger the IQAF value, the better the image qualityhe. The IQAF value of Fig. 1 d) in Fig. 1[13] is the biggest, so the its qality is the best.

\section{B. IQAF Is Able to Assess Quality Degradation as Well as Quality Improvement of An Image}

Fig. 1 b) is the result after Fig. 1 a) passing thruogh certain system and the qulity of Fig. 1 b) is worse than Fig. 1 a) because the $\boldsymbol{C A F}$ value of Fig. 1 b) , i.e. 11.9648, is less than Fig. 1 b), i.e. 14.2346. Fig. 1 d) is the result after Fig. 1 c) passing thruogh certain system and the qulity of Fig.1 d) is better than Fig. 1 c) because the $\boldsymbol{C A F}$ value of Fig. $1 \mathrm{~d}$ ), i.e. 17.9135, is bigger than Fig. 1 c), i.e. 16.5018.

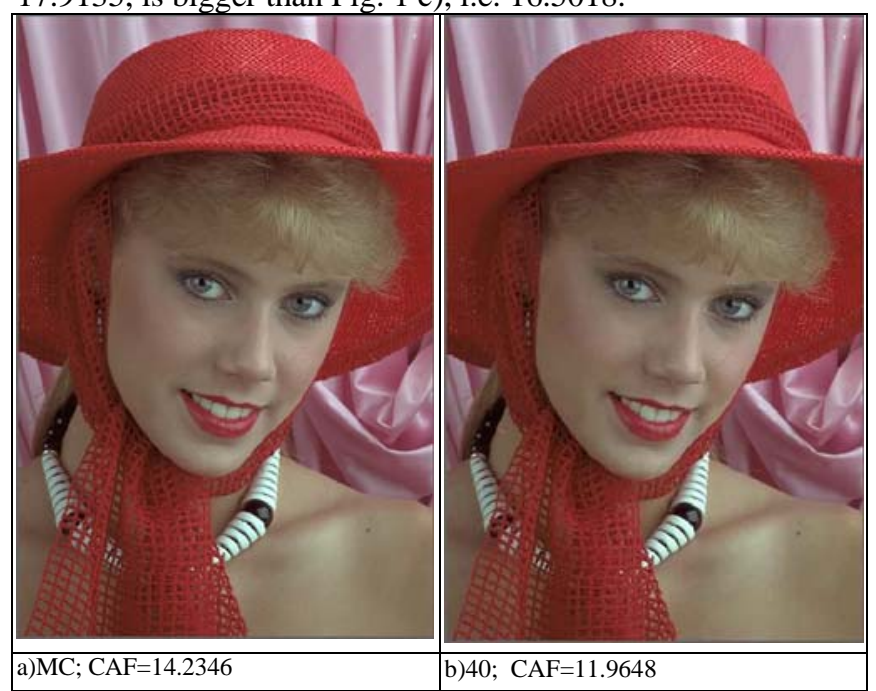




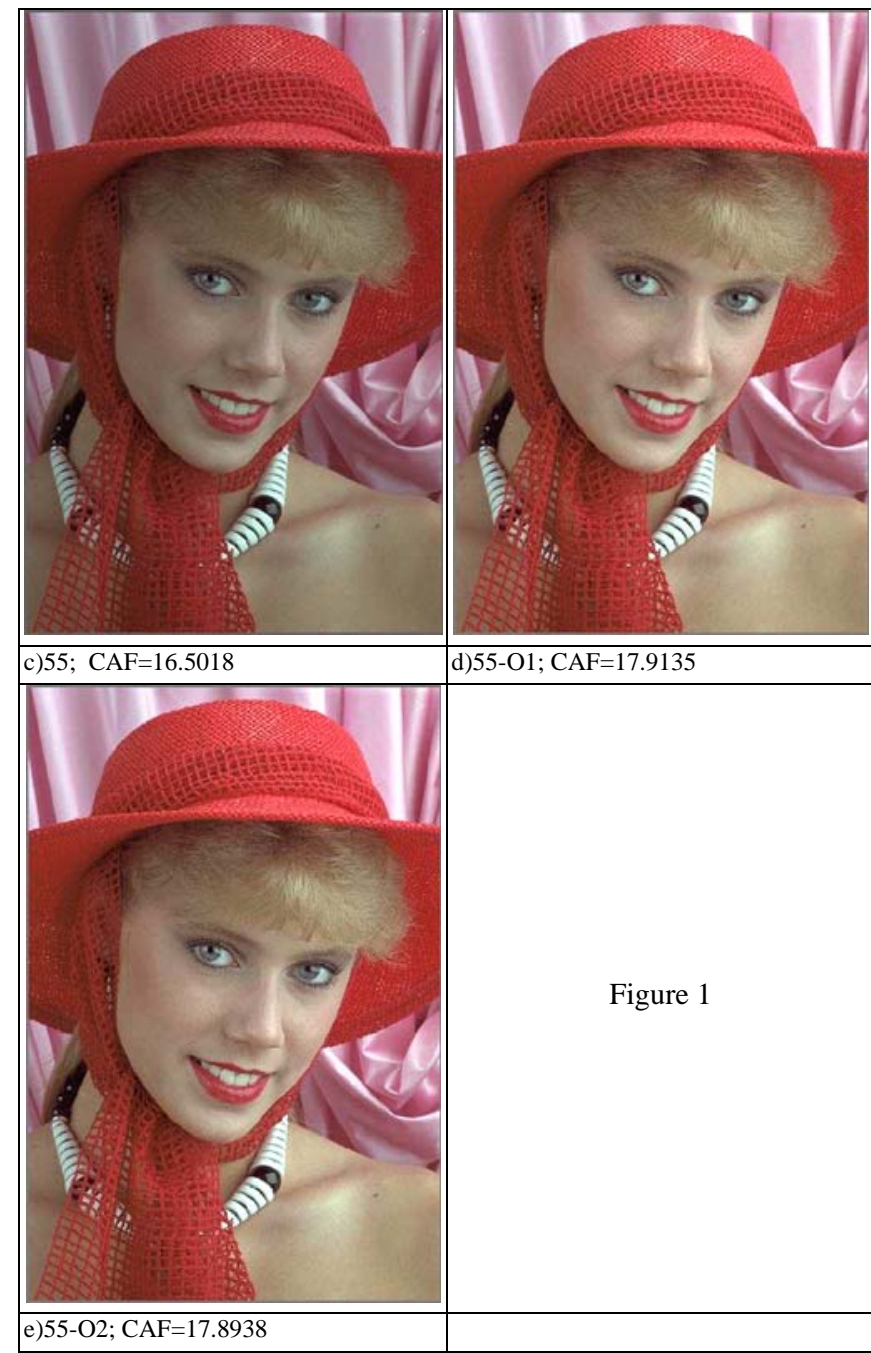

\section{IQAF Is Able to Assess Single Image Quality}

There is a single image such as Fig. 1 c). The $\boldsymbol{C A F}$ of this image is noted as the $\boldsymbol{C A F - O}$. How to judge if its quality is the optimal or not? We apply a disturbance transformation and compute the $\boldsymbol{C A F}$ of the resulting image to see if the $\boldsymbol{C A F}$ of the resulting image is bigger than the former. The disturbance transformation is as follows.

$$
T(i, x, y)=k \frac{O(i, x, y)-0}{2 * A L}, \quad i=0,1,2
$$

and

$$
T(i, x, y)=\left\{\begin{array}{ccc}
255, & T(i, x, y)>255 \\
0, & T(i, x, y)<0 &
\end{array} \quad i=0,1,2\right.
$$

where $\mathbf{i}=\mathbf{0 , 1 , 2}$ represents three color components of the red, the green and the blue. $\boldsymbol{k}$ was called an expansion or a contraction factor, here let $\boldsymbol{k}=255$. $\boldsymbol{O}(\boldsymbol{i}, \boldsymbol{x}, \boldsymbol{y})$ and $\boldsymbol{T}(\boldsymbol{i}, \boldsymbol{x}, \boldsymbol{y})$ represents chroma value of a pixel point $(x, y)$ before and after transformation at $\boldsymbol{i}^{\text {th }}$ channel, respectively. $\boldsymbol{A L}$ is the average luminance of the image.

If the $\boldsymbol{C A F}$ of the resulting image is bigger than the former, then the image quality can be improved. At this time, the disturbance transformation can be done continuously till the $\boldsymbol{C A F}$ of the resulting image is not changed. The last $\boldsymbol{C A F}$ was called $\boldsymbol{C A F - E}$ and correspond to the optimal quality image. The following formula

\section{$R Q D=A b s(E C A F-O C A F) / E C A F$}

is called a relative quality distance. The symbol $\boldsymbol{A b s}(\cdot)$ is called absolute operator. The formula can be used to measure the relative distance from the image to its optimal quality image. An image with $\boldsymbol{R Q D}$ of zero is an optimal quality image. Fig. $1 \mathrm{~d}$ ) is the result that the Fig. $1 \mathrm{c}$ ) is optimized. $\boldsymbol{R Q D}$ of Fig. $1 \mathrm{c}$ ) is $7.88 \%$.

\section{IQAF Can Resolve the Quality Difference That Can not} Be Resolved by Human Vision

The qualities of Fig. $1 \mathrm{c}$ ), d) and e) are difficult to be resolved by human vision, especially the quality difference between Fig. $1 \mathrm{~d}$ ) and e). Use of $\boldsymbol{C A F}$ values can resolve the qualities among them. The first is Fig. $1 \mathrm{~d}$ ), and the second is Fig. 1 e). The last one is Fig. $1 \mathrm{c}$ ). On the basis of the above mentioned, we can say that a machine vision in assessing an image quality can be constructed by means of formula (1). This machine vision assessing image quality originates from human vision and is superior to it.

\section{ACKNOWLEDGMENT}

This work is supported by National Natural Science Foundation of China (Grant No.60975008), gratefully.

Corresponding author: Z. X. Xie, Email: bmezxxie@163.com

The first author: Bo Zhang, Email: cqipcmail@163.com

\section{REFERENCES}

[1] Z. X. Xie, Z. F. Wang, X. L. Xiong, Q. Hu. Color Image Quality Assessment Based on Noise Model of Human Vision Perception and Color Image Quality Optimization. Journal of Image and Graphics. 15(10): 1454-1464, 2010.

[2] M. J. Chen, A. C. Bovik. Fast Structural Similarity Index Algorithm. J Real-Time Image Proc. 6(4):281-287. 2011.

[3] Z. Wang and A. C. Bovik, "Modern Image Quality Assessment”, Morgan \& Claypool Publishers, San Rafael, CA USA, 2006

[4] Y. Wang, W. Liu and R. Wang. "Color Image Quality Assessment Based on Quaternion Singular Value Decomposition”. Proceedings of CISP'08 Congress on Image and Signal Processing. Vol. 3, pp. 433-9, 2008.

[5] S. Suresh, R. V. Babu and H. J. Kim. "No-Reference Image Quality Assessment Using Modified Extreme Learning Machine Classifier”. Applied Soft Computing, No. 9, pp. 541-52, 2009.

[6] R. V. Babu, S. Suresh and A. Perkis. "No Reference JPEG-Image Quality Assessment Using GAP-RBF. Signal Processing. No. 87, pp. 1493-1503, 2007.

[7] S. Suthaharan. "No-Reference Visually Significant Blocking Artifact Metric for Natural Scene Images”. Signal Processing. No. 89, pp. 1647-52, 2009. 
[8] H. R. Sheik, A. C. Bovik and L. Cormack. "No-reference quality using Natural Scene Statistics: JPEG2000. IEEE Transaction on Image Processing”. 14(11): 1918-27, 2005.

[9] K. Seshadrinathan and AC. Bovik. Automatic Prediction of Perceptual Quality of Multimedia Signals-A Survey. Multimed Tools Appl. 2011; 51:163-186.

[10] Y. Y. Shi, Y. D. Ding, R. R. Zhang, J. Li. Structure and Hue Similarity for Color Image Quality Assessment. 2009 International Conference on Electronic Computer Technology, Page(s): 329 - 333, 2009.

[11] Z.X. Xie, Z.F. Wang, X.F. Lv. Single image quality assessment and creation of the best quality image. Proceedings of 2nd International Conference on Information Science and Engineering, ICISE2010 2010:4022-4025, 2010

[12] Z. X. Xie, Z. F. Wang, Y. H. Liu, et al. The Theory of Gradually Flattening Gray Spectrum. Chinese Journal of Medical Physics, 2006, 23(6): 15-17, 36.

[13] http://live.ece.utexas.edu/research/quality/subjective.htm. 